Einführung in das Schwerpunktthemo

\section{Determinanten des Stadtverkehrs}

$\mathrm{D}$

Von Heike Plämig modernen Gesellschaften - trotz des Wissens um die Umwelffolgen - ständig weiter. Die Zunahme dieser Mobilitätsbediurfnisse führt $\mathrm{zu}$ einem Wachstum des Verkehrs und geht mit negativen Begleiterscheinungen für Mensch und Umwelt einher. Luft- und Lärmemissionen, Unfallfolgen sowie Verbrauch, Zerstörung und Zerschneidung von Natur und Landschaft sind nur einige von ihnen.

Die Verteilung und Konzentration der Schadstoffe wird durch die Verkehrsströme und die Verkehrsdichte bestimmt und zeigt räumliche Unterschiede. Gerade in den Städten kann sich niemand den Folgen des immer weiter steigenden motorisierten Verkehrs entziehen (vgl. Stichwort: Verkehrsentwicklung in den Städten). Lärmbelästigungen und Luftverschmutzung, ,stehende Blechlawinen“ und der tägliche Überlebenskampf im Verkehr sind für jeden Einzelnen direkt spür- und erlebbar.

Die Handlungsmuster von Unternehmen, Haushalten und öffentlicher Hand laufen jedoch entgegen der Erwartung; nicht das Problem selbst wird zum Ansatz der Lösungsstrategie gemacht, sondern es finden Ausweichreaktionen statt: Die öffentliche Hand baut (extensiv) die Verkehrswege aus. Unternehmen und Haushalte nehmen die sinkende Wohn- und Aufenthaltsqualität sowie die abnehmende Funktionsfähigkeit der Verkehrsinfrastruktur in den Städten zum Anlass, Standortentscheidungen gegen die (Kern-)Stadt zu richten. Als Folge kommt es zu neuen Konstellationen der städtischen Nutzungen und zu mehr Verkehr.

Konsum zwischen

\section{Ethik und Erlebnis}

\author{
ist das Thema \\ des Informationsdienstes \\ Ökologisches \\ Wirtschaften 1/2000
}

Wenn Sie potenzielle Beiträge

haben, wenden Sie sich bitte an die Redaktion.
Für die Verkehrserzeugung und die veränderte Stadtentwicklung werden vor allem gesellschaftliche und wirtschaftliche Modernisierungsprozesse verantwortlich gemacht, die in zunehmender Individualisierung, zeitlicher Flexibilisierung und räumlicher Fragmentierung zum Ausdruck kommen.

\section{Gesellschaftliche Veränderungsprozesse}

Gesellschaftliche Modernisierungsprozesse führen zu neuen individuellen Lebensstilen, mit starken Einflüssen auf Mobilitätsnachfrage und -organisation. Eine wichtige Rolle spielen vor allem die Veränderungen der Arbeitswelt und anderer sozialer Prozesse: Frauen nehmen zunehmend am Erwerbsleben teil, es gibt immer mehr Personen, die Teilzeitarbeitsplätze haben, und Menschen, die in mehreren Arbeitsverhältnissen beschäftigt sind. Je nach individueller Lebenslage will die Erwerbsarbeit auch mit anderen Aktivitäten (Freizeitgestaltung, Familienarbeit, Ehrenamt) vereinbart sein. Ein großer Bevölkerungsanteil verfügt über viel Freizeit (Stichworte: kürzere Arbeitszeiten, hohe Arbeitslosigkeit, mehr Rentner). Nicht umsonst ist der Freizeitverkehr derjenige mit den höchsten Wachstumsraten.

Auch die zeitliche Flexibilisierung von Arbeitsund Betriebszeiten, beispielsweise das neue Arbeitszeitmodell der Volkswagen AG oder die veränderten Ladenöffnungszeiten, und die Auflösung des Normalarbeitsverhältnisses führen zu starken Disparitäten in den Lebensstilen und deren individueller Gestaltung, die sehr unterschiedliche Ansprüche an das Verkehrssystem stellen: Günstig, schnell, flexibel, bequem lauten hier Schlagworte. Je ausdifferenzierter sich diese Ansprüche darstellen, desto schlechter werden die Ausgangsbedingungen für die Massenver-

\section{Trennung von Wohnen und Arbeif}

Von grundlegender Bedeutung für das Verkehrsvolumen sind die im Vorfeld getroffenen langfristigen Entscheidungen zum Wohnort und zur Arbeitsstelle. Zunehmend fällt die Wahl auf Wohn- und Arbeitsorte außerhalb der Kernstädte. Wohnstandorte und Unternehmensansiedlunkehrsmittel Bus und Bahn. gen (Arbeitsorte) am Rand der Kernstädte werden oft als attraktiver wahrgenommen als das Leben und Arbeiten in der Stadt. Die abgewanderten Einwohner sind meist weiterhin in den Kernstädten beschäftigt und nehmen längere Arbeitswege in Kauf. Sie werden zu Pendlern. Mit weiter steigendem Wohneigentum wird die Mobilitätsnachfrage zunehmen, da der Wohnort in der Regel seltener gewechselt wird als der Arbeitsplatz. Gut ausgebaute Straßen, die Massenmotorisierung und ein attraktives öffentliches Verkehrsangebot begünstigen dieses Mobilitätsverhalten, weil damit die tägliche Überbrüickung größerer Entfernungen zwischen Wohn-, Arbeits-, Versorgungs- und Freizeitort möglich bzw. erleichtert wird.

Die veränderten Mobilitätsbedïrfnisse sind auch auf Knappheiten am Wohnungsmarkt und auf die unterschiedliche Ausstattung der Regionen mit Arbeitsplätzen zurïckzufuihren: Häufig ist es nur möglich, entweder eine gute Stelle oder eine passende Wohnung in einer Region zu finden. Bindungen der Menschen an Familie, Partner oder Partnerin oder Haus (Wohnen im Grünen) sind weitere Gründe dafür, dass Erwerbstätige weite Arbeitswege in Kauf nehmen.

Unter zeitlichen und räumlichen Aspekten finden diese neu organisierten privaten Lebensentwürfe ihren Ausdruck in der zunehmenden Anzahl von Einpendlern in die Kernstadt, in steigenden Distanzen bei den übrigen Aktivitäten, und in der Zuwanderung von Bevölkerung in kleine Gemeinden ohne ausreichende lokale Verkehrsinfrastruktur. Im Zuge dieser Entwicklung kommt es zu einer generellen Bedeutungszunahme des motorisierten Individualverkehrs, verbunden mit einer weiter steigenden privaten Motorisierung. Die Suburbanisierung löst somit eine mobilitätssteigernden Trend aus und lässt die zurückgelegten Distanzen stetig ansteigen.

\section{Wirtschaftliche Veränderungen}

Vor dem Hintergrund der europäischen Integration, einer wachsenden räumlich-funktionalen Arbeitsteilung und eines verschärften Wettbewerbs haben sich die ökonomisch-technischen und politischen Rahmenbedingungen der Unternehmen stark verändert. Der wirtschaftliche Strukturwandel und die neuen Produktions- und Logistikstrategien haben zu starken Verschiebungen von Verkehrswachstum und Verkehrsanteilen innerhalb und zwischen den einzelnen Verkehrsträgern im Güterverkehr geführt. 
Von Heike Flämig

n den größeren Städten umfosst der Anteil der Verkehrs-

fläche an der Gesamiflüche 15 bis 20 Prozent. Die Viel. zahl und Größe der Verkehrsflächen sind weder cus ökolo. gischer noch aus städtebaulicher Sicht zu vertreten. Gleichzeitig treten in den engen Straßenrüumen der Innenstädte Nutzungskonflikte zwischen dem motorisierten Individualverkehr (MIV), dem öffentlichen Personennahverkehr (ÖPNV), den Anwohnern und dem Wirtschaftsverkehr ouf. Rund 65 Prozent der Bevölkerung fühlen sich durch Verkehrslärm belästigt. In vielen Städten sind die Umfeldbelastungen inzwischen so hoch geworden, dass die städtischen Einwohnerzahlen stark rückläufig sind.

Diese spezifisch kommunalen Probleme sind seit der Massenmotorisierung zum großen Teil auf die Autonuzzung zurückzutühren; mit weiter steigender Tendenz. Mit einem Anteil von 48,5 Prozent bei den Stickoxiden und 52,4 Prozent bei Kohlenmonoxid ist der Stroßenverkehr der Houptverursacher der Luftschadstoffemissionen (1). Auch in Großstädten, die in der Regel über einen attrokiveren öffentlichen Verkehr verfügen als Kleinstädte oder lëndli-

\section{Stichwort: Verkehrsentwicklung in den Städten}

che Regionen, beträgt der durchschnittliche Pkw-Anteil an den zurückgelegten Wegen über 50 Prozent (val. Tabelle 1). Die Aufteilung zwischen den Verkehrsträgern (Modal. Split) sagt allein allerdings nichts über die realen ökologischen und soziolen Belastungen aus, die sich erst aus der Multiplikation der spezifischen Verbrouchswerte und Emissionen der benutzten Verkehrsmittel sowie der zurückgelegten Distanzen ergeben.

In den letżen zwei Jahrzehnten ist eine deutliche Zunahme des Akcionsradius zu verzeichnen: Zwischen 1976 und 1997 ist der Verkehrsoufwand je Einwohner um fost ein Viertel ouf rund 12.000 Kilometer pro Person gestiegen. Dabei hat sich die durchschnittliche Anzahl der Wege je Einwohner $(3,5$ Wege) und die dafür aufgewendete Zeit

Tabelle 1: Verkehrsmittelwahl (Wege der Einwohner) in deutschen Städten

\begin{tabular}{|l|c|c|c|c|c|}
\hline Stadt & Jahr & Fulgänger & Fahirad & Offentlicher Verkehr & Motorisierter Individualverkehr \\
\hline Erfurt & 1990 & $39 \%$ & $4 \%$ & $20 \%$ & $33 \%$ \\
Holle/Saale & 1991 & $34 \%$ & $10 \%$ & $20 \%$ & $35 \%$ \\
Hannover & 1990 & $23 \%$ & $16 \%$ & $22 \%$ & $39 \%$ \\
Bremen & 1991 & $21 \%$ & $22 \%$ & $17 \%$ & $39 \%$ \\
München & 1989 & $24 \%$ & $12 \%$ & $24 \%$ & $40 \%$ \\
Freiburg & 1990 & $22 \%$ & $18 \%$ & $16 \%$ & $43 \%$ \\
Kassel & 1990 & $29 \%$ & $6 \%$ & $17 \%$ & $47 \%$ \\
Bochum & 1990 & $27 \%$ & $5 \%$ & $10 \%$ & $57 \%$ \\
\hline
\end{tabular}

Quelle: gsf-Forschungszentrum Umwelt und Gesundheit: Stadtökologie. Berichte aus der ökologischen Forschung. München 1997, S. 32.

Ein wesentlicher Auslöser ist der Güterstruktureffekt, der die Veränderungen auf den Gütermärkten beschreibt. Für den Güterstruktureffekt werden insbesondere der Bedeutungsverlust von Grundstoff- und Montanindustrien, der Bedeutungsgewinn ,hochwertiger" Güter und die Verlagerung von Massenproduktionen in das Ausland verantwortlich gemacht. Gleichzeitig trägt das abnehmende Gütergewicht auch zum Rückgang der Transportkosten an den Gesamtkosten der Güterproduktion und -verteilung bei. Die Entwicklungen in der jüngeren Zeit haben die Bedeutung der Transportkosten als Standortfaktor weiter reduziert. Dazu gehören vor allem der Ausbau der . Verkehrswege (Infrastruktureffekt), die Entwicklung bei den Verkehrstechnologien und die Ordnungspolitik der Europäischen Union (Deregulierungs- und Liberalisierungseffekt) (1). Die hochwertigeren Güter und die neuen Produktionsstrategien von Industrie und Handel erfordern neue Logistikkonzepte (Logistik- effekt). Auf die Schnelllebigkeit der Produkte und den Wunsch vieler Kunden nach kurzen Lieferzeiten reagieren die Unternehmen vor allem durch verkïrzte Produktions- und Lieferrhythmen (Just-in-Time). Die zeitliche Flexibilisierung spiegelt sich in der dynamischen Marktentwicklung der Kurier-, Express- und Paketdienste (KEP-Branche) wider.

Im Rahmen der räumlichen Flexibilisierung werden im großen Umfang Tätigkeiten und Funktionen ausgelagert (Outsourcing), wodurch es zur Zunahme von Arbeitsteilung, räumlicher Verflechtung und Verkehrsaufwand kommt. Außerdem werden neue hochflexible Produktionsstätten aufgrund fehlender Flächenreserven und hoher Bodenpreise in den Innenstädten außerhalb der Kernstädte angesiedelt.

Mit den veränderten logistischen Anforderungen und der Suburbanisierung der Arbeits- und Produktionsstätten verbinden sich unterschiedliche Reaktionsmöglichkeiten der Verkehrsträger, ent-
(82 Minuten) koum erhöht (2)

Die Zuwöchse werden nahezu ousschlieilich im Außerorts. bereich erbracht, während dos Wachstum der Kfz-Fahrleistung in den Stödten stagniert. Das Verhältnis von Innerorts- zu Auferortsverkehr beträgt ca. 27 zu 73 Prozent. Neben dem Zuwachs der (transitbedingten) Fahrleistungen ouf Bundesoutobahnen und den anhaltenden Suburbanisierungsprozessen ist diese Entwicklung auch auf cie Staus im innerstädtischen Bereich zurïckzuführen.

Durch diese Entwicklung besitzen die Städte hohe Anteile an den Fernverkehren, da sich das Verkehrsaufkommen in Abhängigkeit von der Verteilung der Bevölkerung räumlich dorstell: Viele Fernverkehre haben ihre Quelle oder ihr Ziel in Str̈dten und Verdichtungsröumen. Dies gilt gleichermaßen für den Wirtschaftsverkehr, dessen Anteil an den Fohrleistungen in den Stödten bei rund 30 Prozent liegt; punktuell ist er sogar noch höher. Der Verkehr ist daher keineswegs - wie gelegentlich suggeriert wird - immer störker Ausdruck von Fernverbindungen und globalen Zusommenhängen: Personen- und Güterverkehr bestehen zu erheblichen Toilen aus Nahverkehr, d.h. aus Verkehr in Distonzen unter 50 bzw. $75 \mathrm{~km}$ sowie Binnenverkehren. Reine Durchgangs- oder Transitverkehre besitzen sowohl im Personenverkehr als auch im Güterverkehr sehr niedrige Anteile und werden oft überschötzt.

\section{Anmerkungen}

(1) Vorläutige Werte für 1996; ohne Land, Forst- und Bouwirtschaft. Vgl. Bundesverkehrsministerium (BMV) (Hrsg.): Verkehr in Zohlen. Bonn 1998, S. 285. (2) Fü die Zahlenangaben vgl. BMV (Hrsg.): Verkehr in Zehlen. Bonn 1998, S. 227 und 159.

sprechend den sogenannten Verkehrswertigkeiten (2): Schnelligkeit, Netzbildung und Flexibilität. Während die Schiene und das Binnenschiff angestammte Märkte verlieren, fallen dem flexiblen Lkw und der Luftfracht neue Potenziale zu. Das Zusammenwirken mit niedrigen Transportkosten führt $\mathrm{zu}$ Ineffizienzen, die sich in ungenutzten Ladekapazitäten, vor allem bei den innenstadtbezogenen Touren, zeigen.

\section{- Schlussfolgerungen}

Um dem Verkehrswachstum zu begegnen, werden in der Verkehrsplanung und -politik derzeit verschiedene Handlungsstrategien als Wege zu einer nachhaltigen Verkehrsstruktur diskutiert, vorrangig auf der Ebene der Verkehrsvermeidung, der Verkehrsverlagerung auf weniger umweltschädliche Verkehrsmittel und der verträglicheren Abwicklung (vgl. Kasten: Politikziele und Planungsstrategien im Stadtverkehr). 


\section{Politikziele und Planungsstrategien im Stadtverkehr}

Die Sicherstellung der Mobilität für alle Bevölkerungsgruppen und alle Funktionen in der Stadt sowie die stadtund umweltverträgliche Gestaltung der Mobilität sind anerkannte Ziele der Stadtverkehrspolitik von Bund, Ländern und Gemeinden (3). Angesichts der Belastungsgrenzen von Stadtraum und Umwelt sowie vor dem Hintergrund der Kapazitätsgrenzen der Verkehrsinfrastruktur kommt es bei weiterhin steigender Mobilitätsnachfrage zumindest in den großen Städten zu verschärften Konflikten zwischen funktionalen und städtebaulichen Ansprüchen an den Verkehr.

Die offensichtlichen Probleme des wachsenden Verkehrs dazu beigetragen, dass neben die Sicherstellung von Leichtigkeit und Flüssigkeit des Straßenverkehrs städtebauliche, soziale und ökologische Ziele als neue Planungsziele traten. Derartige Ziele sind Bestandteil von Handlungskonzepten von Parteien, des Deutschen Bundestages, von Landesregierungen, Kommunen und auch von Gewerkschaften und Unternehmensleitlinien.

Aus dem verkehrspolitischen Anspruch, eine städtebaulich, sozial bzw. ökologisch orientierte Verkehrsentwicklung in Gang zu setzen, werden folgende Handlungsstrategien abgeleitet:

- Der motorisierte Verkehr soll vermieden bzw. verringert werden (Wegfall oder Verkürzung von Fahrten).

- Der öffentliche und der nicht-motorisierte Verkehr sollen gefördert werden.

- Nicht vermeidbarer motorisierter Verkehr soll verlagert werden (im Personenverkehr vom Pkw auf öffentlichen Nahverkehr, Fahrrad oder auf die Füße; im Güterverkehr auf Bahn, Binnenschiff oder Lastenfahrrad).

- Der verbleibende motorisierte Verkehr soll umweltund sozialverträglich abgewickelt werden (z.B. Verringerung der Lärm- und Schadstoffbelastungen und des Enerkungen). tenverkehre),

- aller Verkehrszwecke, ("sektoral"),

("horizontal"), in Städten haben spätestens seit Anfang der 80-er Jahre

gieverbrauchs durch technische Maßnahmen; Erhöhung der Verkehrssicherheit durch Geschwindigkeitsbeschrän-

Dabei erhält (nur) ein konsequentes Bemühen um verkehrssparsame Rahmensetzungen in anderen Sektoren (z.B. in der Raumplanung) die Option auf ein entsprechendes Umstevern im Verkehrsbereich: Zwar können indirekt wirksame Konzepte eine am Umweltverbund und an geringeren Distanzen orientierte Verkehrspolitik und -planung nicht ersetzen. Sie können diese aber unterstützen.

\section{Infegrierfe Planung notwendig}

Demnach reicht eine lediglich auf das Verkehrssystem selbst gerichtete Strategie nicht aus. Für eine erfolgreiche Umsetzung einer ökologieorientierten Verkehrsplanung und -politik wird daher heute eine integrierte Planung aller Elemente des Verkehrssystems und aller Planungsebenen als dringend erforderlich angesehen. Eine integrierte Verkehrsplanung berücksichtigt sowohl die unterschiedlichen Anforderungen an die Gestaltung der Verkehrssysteme, als auch die Auswirkungen auf die Arbeitsbedingungen und die soziale Situation der Beschäftigten. Im einzelnen geht es um die Integration - aller Verkehrsarten (Personen-, Güter- und Nachrich-

- aller Verkehrsmittel und Verkehrsträger (

- der Raum-, Wirtschafts-, Sozial- und Umweltplanung

- von benachbarten Gebietskörperschaften

- von benachbarte Planungsebenen („vertikal") und

- von verschiedenen Maßnahmenkomplexen (4).

Die vorangestellten Ausfïhrungen haben jedoch gezeigt, dass Verkehrswachstum und Verkehrserzeugung häufig auf Entscheidungen außerhalb des Verkehrssystems zurïckzufuihren sind: Veränderte Rahmenbedingungen lassen Individuen und Unternehmen anders als bisher agieren, neue Handlungsmuster entstehen mit einer veränderten Nachfrage und Organisation von Mobilität. Die Bevölkerung, Produktions- und Versorgungsfunktionen zieht aus den Innenstädten an die Verdichtungsränder, wie es die wachsenden regionalen Pendlerbeziehungen und Kaufkraftströme eindrucksvoll belegen und verändern die Bedeutung der Stadt als multifunktionale Einheit (zum Zusammenspiel von Stadt, Ökonomie und Verkehr siehe den Artikel von Heike Flämig in diesem Heft).
Früher ließen Handel, Produktion, Versorgung und Wohnen die Städte prosperieren, leben, sich entwickeln. Heute scheint sich das Verhältnis umgekehrt $\mathrm{zu}$ haben und ins Negative gekippt zu sein. Die gewachsene dichte und kompakte Stadt behindert ein weiteres Innenwachstum; die innerstädtische Verkehrsinfrastruktur scheint nicht mehr in der Lage zu sein, zusätzliche Verkehre aufzunehmen. Bei der Entscheidung über Wahl und Vergabe von Standorten stehen sich öffentliche Planungsinteressen und einzelwirtschaftliche Anforderungen oftmals diametral gegenüber. Vor diesem Hintergrund analysiert Christian Schneider in seinem Beitrag die aktuelle Entwicklung im Einzelhandel.

Jürgen Glaser sucht nach Lösungsmöglichkeiten, die hohen Umweltbelastungen von inner- städtischen Gewerbegebieten zu minimieren. Christian Holz-Rau setzt den Schwerpunkt auf die städtische Nutzung ,Wohnen“.

Die Schwerpunktbeiträge setzen damit vor allem an den jeweiligen Handlungslogiken, -strategien und -möglichkeiten einzelner städtischen Nutzungen an. Dabei werden sowohl aus Sicht der Planung als auch aus Sicht der Unternehmen die jeweils in der theoretischen Diskussion und praktischen Umsetzung befindlichen "Lösungsansätze“ kritisch reflektiert. Das von den einzelnen Autoren vorgestellte Set erhebt keinen Anspruch auf Vollständigkeit. Vielmehr sollen die jeweils vorgestellten Maßnahmen und Konzepte als Anregung dienen, selbst Prozesse einer zukunftsfähigen Stadtund Verkehrsentwicklung anzustoßen.

Allerdings macht ein Großteil der Beiträge auch deutlich, dass es eine Diskrepanz zwischen Wissen und Handeln gibt. Hier setzt der abschließende Aufsatz von Markus Hesse an. Er fordert ein angemesseneres Bild der veränderten Raum-Zeit-Muster und damit eine notwen-dige Erweiterung der Forschungsperspektive um Aspekte aus der Lebensstil- und der sozial-räumlichen Milieuforschung. So könnte vielleicht doch noch eine zielgerichtete erfolgreiche Auflösung der Verkehrsspirale realisiert werden.

\section{Anmerkungen}

(1) Zu nennen sind hier vor allem der Abbau von Handelshemmnissen allgemein und der Abbau der relativ hohen Regelungsdichte im Verkehrsgewerbe im Besonderen, durch Freigabe von Torifen und Kontingenten, Aufhebung von Marktzugangsbeschränkungen, Neuregelung bzw. Freigabe der Kabotage.

(2) Voigt, F.: Verkehr. 3 Bde. Berlin 1973.

(3) Vgl. beispielhaft Ministerium für Stadtentwicklung und Verkehr des Landes Nordrhein-Westfalen: Gesamtverkehrsplan Nordrhein-Westfalen, Düsseldorf 1990. Deutscher Städtetag: 10-Punkte-Programm zum Stadtverkehr 1992. EG-Kommission: Weißbuch Verkehr und Umwelt.

Brüssel 1992.

(4) Landtag Nordrhein-Westfalen (Hrsg.): Teilbericht der Enquête-Kommission Zukunft der Mobilität. Integrierte Gesamtverkehrsplanung in NRW. Drucksache 12/3246. Düsseldorf 1998.

Die Autorin
Heike Flämig ist Leilerin des Forschungsfeldes Stadt-
entwicklung, Planung, Verkehr des Insituts für öko-
logische Wirtschaftsforschung
Kontakt: 10̈W, Potsdamer Str. 105, 10785 Berlin.
Tel. 030/884594-19, Fax 030/8825439,
E-mail: Heike.Fluemig@ioew.de


(c) 20I0 Authors; licensee IÖW and oekom verlag. This is an article distributed under the terms of the Creative Commons Attribution Non-Commercial No Derivates License (http://creativecommons.org/licenses/by-nc-nd/3.o/), which permits unrestricted use, distribution, and reproduction in any medium, provided the original work is properly cited. 